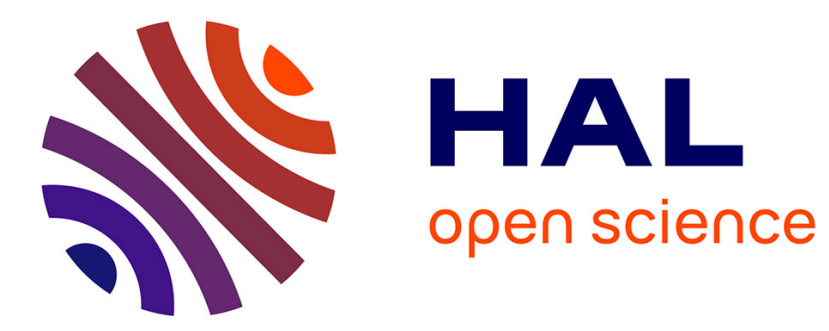

\title{
2D Legendre Moments-Based Visual Control
}

Aurelien Yeremou Tamtsia, Youcef Mezouar, Philippe Martinet, Haman

Djalo, Emmanuel Tonye

\section{To cite this version:}

Aurelien Yeremou Tamtsia, Youcef Mezouar, Philippe Martinet, Haman Djalo, Emmanuel Tonye. 2D Legendre Moments-Based Visual Control. Applied Mechanics and Materials, 2012, 162, pp.487-496. 10.4028/www.scientific.net/AMM.162.487 . hal-02463477

\section{HAL Id: hal-02463477 \\ https://hal.inria.fr/hal-02463477}

Submitted on 1 Feb 2020

HAL is a multi-disciplinary open access archive for the deposit and dissemination of scientific research documents, whether they are published or not. The documents may come from teaching and research institutions in France or abroad, or from public or private research centers.
L'archive ouverte pluridisciplinaire HAL, est destinée au dépôt et à la diffusion de documents scientifiques de niveau recherche, publiés ou non, émanant des établissements d'enseignement et de recherche français ou étrangers, des laboratoires publics ou privés. 


\title{
2D Legendre Moments-based Visual Control
}

\section{Aurelien Yeremou Tamtsia,a, Youcef Mezouar ${ }^{1, b}$, Philippe Martinet ${ }^{1, c}$, Haman Djalo ${ }^{2, \mathrm{~d}}$ and Emmanuel Tonye ${ }^{2, \mathrm{e}}$}

\author{
${ }^{1}$ Clermont Université, UBP, UMR CNRS 6602, LAboratoire des Sciences et Matériaux pour \\ l'Electronique et d'Automatique, BP 10448, F-63000 Clermont-Ferrand, France \\ ${ }^{2}$ Université de Yaoundé I, LAboratoire d'Electronique et du Traitement du Signal, ENSP, BP 8390, \\ Yaoundé- Cameroun \\ aYeremouTamtsia@univ - bpclermont.fr, ${ }^{b}$ youcef.mezouar@lasmea.univ-bpclermont.fr, \\ cPhilippe.Martinet@irccyn.ec - nantes.fr, ${ }^{c}$ haman_djalo@yahoo.com, ${ }^{\text {et }}$-nyee@hotmail.com
}

Keywords: orthogonal moments, visual servoing

\begin{abstract}
Among region-based descriptors, geometric moments have been widely exploited to design visual servoing schemes. However, they present several disadvantages such as high sensitivity to noise measurement, high dynamic range and information redundancy (since they are not computed onto orthogonal basis). In this paper, we propose to use a class of orthogonal moments (namely Legendre moments) instead of geometric moments to improve the behavior of moment-based control schemes. The descriptive form of the interaction matrix related to the Legendre moments computed from a set of points is first derived. Six visual features are then selected to design a partially-decoupled control scheme. Finally simulated and experimental results are presented to illustrate the validity of our proposal.
\end{abstract}

\section{Introduction}

Shape descriptors have been widely exploited in the computer vision community. They can generally be ranked as region based or contour-based descriptors. Contour-based descriptors only capture the contour information while region-based descriptors can also capture the interior content of the shape and contain thus more information. Among region-based descriptors, moments are certainly the most popular. They have been first introduced in the computer vision community by $\mathrm{Hu}$ [1] and they include geometric moments [1], invariant moments [1], Legendre moments [2], Zernike moments [2], [3] and Tchebyshev moments [4], [5]. In the context of shape analysis, moments are defined as the projections of a function defining the object onto a set of functions characteristic of the considered moments. Geometric moments are certainly the most popular global descriptors for shape description when dealing with computer vision applications. However, they present a number of disadvantages. They are highly sensitive to noise and they exhibit very high dynamic range (the moments computed have large variation in the dynamic range of values for different orders; this may cause numerical instability). Furthermore, they contain a large amount of redundant information since the projection basis is not orthogonal. Moments involving orthogonal basis functions, such as the Legendre and Zernike polynomials do not suffer from these drawbacks. They were first exploited in the context of image analysis by Teague [2]. Teh and Chin reported in [6], that orthogonal Legendre moments can be used to represent an image with minimum amount of information redundancy. Properties to assess by image descriptors include invariance with respect to geometric transformations (such as translation, rotation, scaling), stability to noise and small local deformations. The major drawback of orthogonal moments is the lack of native scale invariance. To solve this problem, a normalization process is often used to achieve scale invariance. 
Chong et al. [7] proposed recently a method based on the properties of pseudo-Zernike polynomials to derive the scale invariants of pseudo-Zernike moments. A similar approach was proposed in [8] to design both translation and scale invariants of Legendre moments. A refined method was also proposed by Hosny in [9]. A new set of rotationally Legendre moments invariants was recently proposed by Hosny in [10]. A large amount of works has been done in geometric moments-based visual servoing while, at our knowledge, our work is the first to address the case of orthogonal moments-based visual servoing. The visual servoing schemes proposed by Chaumette in [11] and Tahri et al. in $[12,13]$ are closely related to the work presented in this paper. In [11], the determination of the descriptive form of the interaction matrix related to any geometric image moments is presented. The selections of six visual features (to control six degrees of freedom of a camera) are detailed in $[11,12,13]$. The proposed selections are mainly based on the intuitive meaning of geometric moments and on the invariant visual features computed from geometric loworder image-moments (less than three). In this paper, we propose to exploit orthogonal Legendre moments as visual features and the related descriptive form of the interaction matrix to design a vision-based control scheme. The remaining of this paper is structured as follows. We first give the essential of Legendre moments. We then present the computation of the interaction matrix related to Legendre moments. Simulation and experimental results are presented to validate our approach.

\section{Geometric moments}

Let $f(x, y) \geq 0$ be a real bounded function with support on a compact region $R$. The $(p+q)$-order moments of $f$ are defined as [14]:

$m_{p q}=\iint_{R} x^{p} y^{q} f(x, y) d x d y$

In terms of geometrical moments, the orientation of the principal axes $\Phi$ can be computed as follows:

$\phi=\frac{1}{2} \tan ^{-1}\left(\frac{2 \mu_{11}}{\mu_{20}-\mu_{02}}\right)$

Where the centered moments $\mu_{\mathrm{pq}}$ are computed with respect to the object centroid $\left(\mathrm{x}_{\mathrm{c}}, \mathrm{y}_{\mathrm{c}}\right)$ :

$\mu_{p q}=\iint_{R}\left(x-x_{c}\right)^{p}\left(y-y_{c}\right)^{q} f(x, y) d x d y$

The centered moments are known to be invariant to 2D translational motions. Note that if the image is not sufficiently textured, weighted moments of the image cannot be exploited to compute the rotation angle (2). In this case, it is possible to use higher order moments of the binary image. The use of higher order moments to provide rotational invariants was extensively discussed in the literature $[1,15,16]$. The method of principal axes described by $\mathrm{Hu}[1]$ allows to obtain rotational relations among the same order moments terms (note that using second order moments we obtain equation (2) which was largely exploited in the context of geometric moments-based visual servoing). For instance, the third order moment can be used to determine the orientation angle of a target:

$$
\beta=\arctan \left(\frac{\varphi_{21}+\varphi_{03}}{\varphi_{12}+\varphi_{30}}\right)
$$


It is known that high order moments are more discriminative (for a given object) than low order moments but geometrical moments are also more sensitive to measurements noises. Using Legendre moments, we are not restricted to use only low order moments since they are less sensitive to noise measurement than geometric moments.

\section{Legendre moments}

Legendre polynomials $\mathrm{P}_{\mathrm{n}}(\mathrm{x})$ are defined as:

$P_{n}(x)=\sum_{k=0}^{n} a_{n k} x^{k}$

Where the Legendre coefficients $\mathrm{a}_{\mathrm{nk}}$ are given by:

$a_{n k}=(-1)^{\frac{n-k}{2}} \frac{1}{2^{n}} \frac{(n-k) !}{[(n-k) / 2] ![(n+k) / 2] ! k !}$

With $\mathrm{n}-\mathrm{k}=$ even. Legendre moments $\lambda_{\mathrm{pq}}$ are defined from Legendre polynomials as [2]:

$\lambda_{p q}=c_{p q} \int_{-1}^{1} \int_{-1}^{1} P_{p}(x) P_{q}(y) f(x, y) d x d y$

(7)

With $\quad c_{p q}=\frac{(2 p+1)(2 q+1)}{4}$

Similarly than for geometric moments, translational invariants can be obtained by computing central Legendre moments:

$\varphi_{p q}=c_{p q} \int_{-1}^{1} \int_{-1}^{1} P_{p}\left(x-x_{c}\right) P_{q}\left(y-y_{c}\right) f(x, y) d x d y$

Where the intensity centroid can be expressed in terms of Legendre moments as:

$x_{c}=\left(\frac{c_{00}}{c_{10}}\right)\left(\frac{L_{10}}{L_{00}}\right) \quad y_{c}=\left(\frac{c_{00}}{c_{01}}\right)\left(\frac{L_{01}}{L_{00}}\right)$

Chong et al. [8] showed that the general form of two-dimensional Legendre central moments, in terms of Legendre moments, can be obtained as follows:

$$
\begin{aligned}
& \phi_{p q}=\sum_{n=0}^{p} \sum_{m=0}^{q} \frac{c_{p q}}{c_{(p-n)(q-m)}} v_{p(p-n)} k_{q(q-m)} \lambda_{(p-n)(q-m)} \\
& v_{p p}=1, v_{p(p-n)}=\tau_{p-n}\left[\sum_{r=1}^{n}\left(\begin{array}{c}
p-n+r \\
r
\end{array}\right)\left(-x_{c}\right)^{r} a_{p(p-n+r)}-\sum_{s=0}^{n-1} v_{p(p-s)} a_{(p-s)(p-n)}\right], \tau_{p-n}=\frac{1}{a_{(p-n)(p-n)}} \\
& k_{q q}=1, k_{q(q-m)}=\tau_{q-m}\left[\sum_{u=1}^{m}\left(\begin{array}{c}
q-m+u \\
u
\end{array}\right)\left(-y_{c}\right)^{u} a_{q(q-m+u)}-\sum_{w=0}^{m-1} k_{q(q-w)} a_{(q-w)(q-m)}\right], \tau_{q-m}=\frac{1}{a_{(q-m)(q-m)}} .
\end{aligned}
$$

Subject to the conditions: $(n-r)$ even, $(n-s)$ even, $n \geq 1,(m-u)$ even, $(m-w)$ even and $m \geq 1$.

\section{Kinematic of 2D Legendre Moments}

Let $\Omega \subset \mathrm{R}^{2}$ be a closed and bounded region of the plane and $\mathrm{f}(\mathrm{x}, \mathrm{y}): \Omega \rightarrow \mathrm{R}$ be an image defined on $\Omega$, the region containing the objects. We are interested in determining the analytical form describing the time variation $\dot{\lambda}_{p q}$ of Legendre moments $\lambda_{\mathrm{pq}}$ as a function of the relative camera object kinematic screw $\mathbf{v}=(v, \omega)$ 


$$
\dot{\lambda}_{p q}=\mathbf{L}_{\lambda_{p q}} \mathbf{v}
$$

where $\mathbf{L}_{\lambda \mathrm{pq}}$ is the interaction matrix related to the Legendre moments $\lambda_{\mathrm{pq}}$. In the following, we will consider planar objects and we exclude the degenerate case where the camera optical center belongs to it. In that case, for any object point $1 / Z=A x+B y+C$ where $Z$ is the depth of the observed point [17] and A, B, C are the plane parameters. If we consider the image moments defined from a set of points or defined by integration on an area in the image, the interaction matrix related to image moments is given by ( see [13])

$$
\begin{aligned}
& L_{m_{i j}}=\left[\begin{array}{llllll}
m_{v_{x}} & m_{v_{y}} & m_{v_{z}} & m_{w_{x}} & m_{w_{y}} & m_{w_{z}}
\end{array}\right]
\end{aligned}
$$

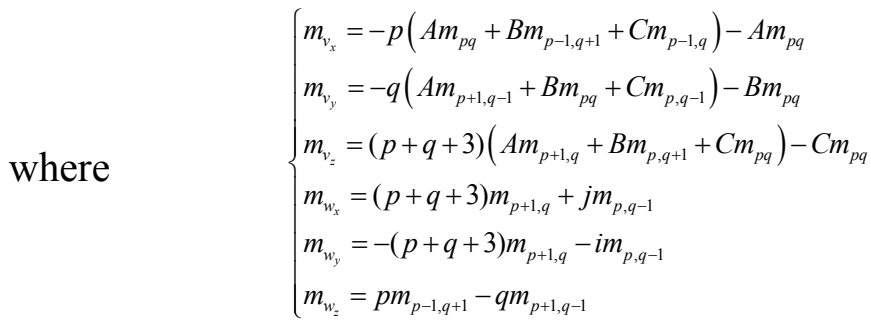

The relationship between conventional image moments and Legendre moments is defined by

$$
\lambda_{p q}=\frac{(2 p+1)(2 q+1)}{4} \sum_{i=0}^{p} \sum_{j=0}^{q} a_{p i} a_{q j} m_{i j}
$$

From (17) and (15), the general form of the interaction matrix related to any Legendre moments can be written as:

$$
\boldsymbol{L}_{\lambda_{p q}}=\frac{(2 p+1)(2 q+1)}{4} \sum_{i=0}^{p} \sum_{j=0}^{q} a_{p i} a_{q j} \boldsymbol{L}_{\boldsymbol{m}_{\boldsymbol{i j}}}
$$

Similarly, if we consider the centered Legendre moments defined by (8) and (9), we obtain:

$$
\begin{aligned}
& L_{\varphi_{p q}}=\left[\begin{array}{llllll}
\varphi_{v_{x}} & \varphi_{v_{y}} & \varphi_{v_{z}} & \varphi_{w_{x}} & \varphi_{w_{y}} & \varphi_{w_{z}}
\end{array}\right] \\
& \varphi_{v_{x}}=-(p+1) \mathrm{A} \varphi_{p q}-p \mathrm{~B} \varphi_{p-1, q+1}+p\left[\left(2 \frac{c_{10}}{c_{00}}-1\right) \mathrm{A} x_{c}+\left(\frac{c_{10}}{c_{00}}-1\right) \mathrm{B} y_{c}\right] \varphi_{p-1, q}+q\left(\frac{c_{01}}{c_{00}}\right) \mathrm{A} y_{c} \varphi_{p, q-1} \\
& \varphi_{v_{y}}=-q \mathrm{~A} \varphi_{p+1 q-1}-(q+1) \mathrm{B} \varphi_{p, q}+q\left[\left(\frac{c_{01}}{c_{00}}-1\right) \mathrm{A} x_{c}+\left(2 \frac{c_{01}}{c_{00}}-1\right) \mathrm{B} y_{c}\right] \varphi_{p, q-1}+p\left(\frac{c_{10}}{c_{00}}\right) \mathrm{B} x_{c} \varphi_{p-1, q} \\
& \text { and } \varphi_{v_{s}}=-\mathrm{A} \varphi_{w_{y}}+\mathrm{B} \varphi_{w_{x}}+(p+q+2) C \varphi_{p, q}+p\left[\left(1+\frac{c_{10}}{c_{00}}\right) C-6\left(\frac{c_{20}}{c_{00}}-\frac{c_{10}}{c_{00}}\right) \mathrm{A} x_{c}\right] x_{c} \varphi_{p-1, q} \\
& +q\left[\left(1+\frac{c_{01}}{c_{00}}\right) C-2\left(\frac{c_{11}}{c_{00}}\right) \mathrm{A} x_{c}+2\left(3 \frac{c_{02}}{c_{00}}-2 \frac{c_{01}}{c_{00}}\right) \mathrm{B} y_{c}\right] y_{c} \varphi_{p, q-1} \\
& \varphi_{w_{c}}=(p+q+3) \varphi_{p, q+1}+p x_{c} \varphi_{p-1, q+1}+(p+2 q+3) y_{c} \varphi_{p, q}-4 p\left[\gamma_{11}+\left(\frac{c_{11}}{c_{00}}-\frac{1}{4}\right) x_{c} y_{c}\right] \varphi_{p-1, q}-4 q\left[\gamma_{02}+\left(\frac{3}{2} c_{00}-1\right) y_{c 0}^{2}\right] \varphi_{p, q-1} \\
& \varphi_{w_{y}}=-(p+q+3) \varphi_{p+1, q}-(2 p+q+3) x_{c} \varphi_{p, q}-q v_{c} \varphi_{p+1, q-1}+4 p\left[\gamma_{20}+\left(\frac{3}{2} c_{00}-1\right) x_{c} x_{c}\right] \varphi_{p-1, q}+4 q\left[\gamma_{11}+\left(\frac{c_{11}}{c_{00}}-\frac{1}{4}\right) x_{c} y_{c}\right] \varphi_{p, q-1} \\
& \varphi_{v_{2}}=p \varphi_{p-1, q+1}-q \varphi_{p+1, q-1}+p\left(1-\frac{c_{01}}{c_{00}}\right) y_{c} \varphi_{p-1, q}-q\left(1-\frac{c_{10}}{c_{00}}\right) x_{c} \varphi_{p, q-1}
\end{aligned}
$$

where

$$
\gamma_{p q}=\frac{\varphi_{p q}}{\lambda_{00}}
$$




\section{Features selection for visual servoing}

In order to control the six degrees of freedom of the camera, we have to select from the previous theoretical results some combinations of features that will insure good properties of the interaction matrix (partially-decoupled structure). In the sequel, we will consider the usual control law [11]:

$$
v_{c}=-\lambda \widetilde{L_{s}^{+}}\left(s-s^{*}\right)
$$

Where $\boldsymbol{v}_{\mathrm{c}}$ is the camera velocity sent to the low level robot controller, $\lambda$ is a positive proportional gain, $\mathrm{s}$ a vector containing the values of the current visual features, $\mathrm{s}^{*}$ their desired values and $\widetilde{L_{s}^{+}}$the pseudo-inverse of an approximation of the interaction matrix related to $\mathrm{s}$.

\section{Potential features to control translational motions:}

In [13], the following visual features had been proposed for the translational motions: $\mathrm{x}_{\mathrm{n}}, \mathrm{y}_{\mathrm{n}}$ and $\mathrm{a}_{\mathrm{n}}$. $\mathrm{a}^{*} \quad$ is Where $\quad a_{n}=Z \cdot \sqrt{\frac{a^{*}}{a}}, \quad x_{n}=a_{n} x_{c}, \quad y_{n}=a_{n} y_{c}$ the desired of the object in the image, and $\mathrm{Z}^{*}$ the desired depth between the camera and the object. If we assume that the desired position of the object is parallel to the image plane (i.e. $A=B=0$ ) and we denote $\boldsymbol{L}_{s}^{\|}$the interaction matrix related to Legendre moments in such position. From (18), we find:

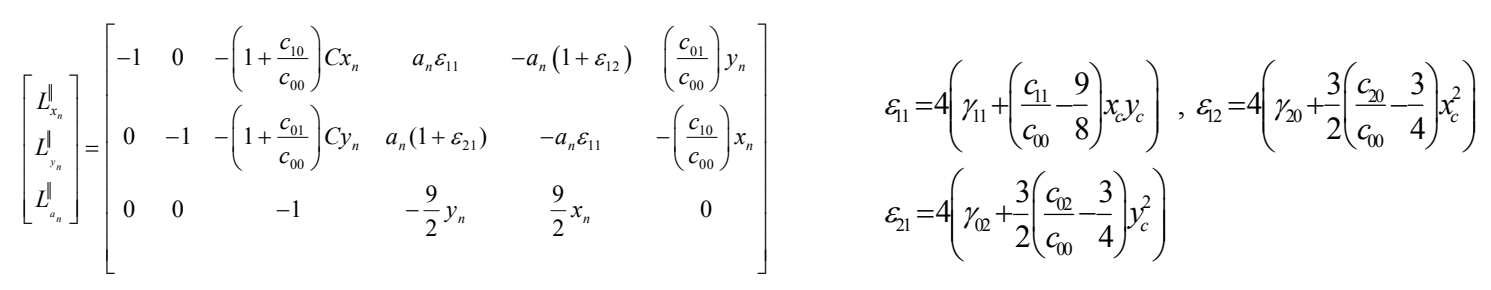

We can note that the block corresponding to the translational DOF is triangular.

\section{Potential features to control rotational motions:}

The orientations of the principal axes are natural features to control the rotational motions. If we substitute centered geometric moments in equations (2) and (4) by Legendre centered moments (9), we obtain:

$$
\alpha=\frac{1}{2} \arctan \left(\frac{2 \varphi_{11}}{\varphi_{20}-\varphi_{02}}\right) \quad \beta=\arctan \left(\frac{\varphi_{21}+\varphi_{03}}{\varphi_{12}+\varphi_{30}}\right)
$$

The interaction matrix related to $\alpha$ and $\beta$ can easily be computed from the previous equations:

$$
\begin{aligned}
& L_{\alpha}=\left[\left(\varphi_{20}-\varphi_{02}\right) L_{\varphi_{11}}-\varphi_{11}\left(L_{\varphi_{20}}-L_{\varphi_{02}}\right)\right] / \Delta \quad L_{\beta}=\left[\left(\varphi_{12}+\varphi_{30}\right)\left(L_{\varphi_{21}}+L_{\varphi_{1}}\right)-\left(\varphi_{21}+\varphi_{03}\right)\left(L_{\varphi_{2}}+L_{\varphi_{0}}\right)\right] / \Delta_{1} \\
& \text { Where } \quad \Delta=\left(\varphi_{20}-\varphi_{02}\right)^{2}+4 \varphi_{11}^{2} \quad \Delta_{1}=\left(\varphi_{12}+\varphi_{30}\right)^{2}+\left(\varphi_{21}+\varphi_{03}\right)^{2}
\end{aligned}
$$

and $\mathrm{L}_{\varphi_{\mathrm{pq}}}$ is the interaction matrix related to $\varphi_{\mathrm{pq}}$ (it can be computed using (19)).

\section{Feature selection:}

In this section, we detail our choice of image features (computed from Legendre moments) to control all six degrees of freedom of the camera. As mentioned before, the area, the coordinates $\left(\mathrm{x}_{\mathrm{n}}\right.$ , $\left.\mathrm{y}_{\mathrm{n}}\right)$ of the center of gravity are natural choices to control the translational DOF. These features computed from geometric moments have already been used in [13] for instance. To control the rotational motions around the optical axis, we can use any of the roll angles provided by the principal axes method. The angle $\alpha$ which is obtained from a combination of second order central 
moments can be an interesting choice and it has been exploited in [11]. Another possible choice is the roll angle $\beta$. This choice seems more suitable since more the moment order is high more it is discriminative. However, all roll angle computed from odd-orders central moments and related to objects with symmetry along $\mathrm{x}$ and/or $\mathrm{y}$ directions, and symmetry with respect to the object centroid have zero values. This implies that $\beta$ cannot be used with symmetrical object since both the numerator and the denominator are equal to zero in this case (refer to equation (23)). We will see in the sequel that by slightly modifing the definition of central Legendre moments it is possible to use any odd-orders moments to compute roll angles with both symmetrical and non-symmetrical objects and thus that it is possible to use the feature $\beta$ to control the camera motions around the optical axis. We need also two supplementary features to control the two remaining rotational DOFs. As a matter of fact, we will improve the decoupling properties of the control law by choosing features which are invariants to translational motions and rotational motions around the optical axis. At this aim, for the two remaining DOF $\mathrm{w}_{\mathrm{x}}$ and $\mathrm{w}_{\mathrm{y}}$, some combinations of Hu's invariants moments can be exploited. However, as noticed in [11] and as for roll angle, the Hu's invariants computed from odd-orders central moments and related to objects with symmetry along $\mathrm{x}$ and /or y directions, and symmetry with respect to the object centroid have zero values. In [11], it was proposed, with geometric moments, to distinguish two cases depending on whether the observed object is symmetrical or not. The author proposed to use:

$$
H_{1}=c_{1}^{2}+s_{1}^{2} \quad H_{2}=c_{2}^{2}+s_{2}^{2} \quad H_{3}=\mu_{20}+\mu_{02}
$$

where $c_{1}=\mu_{20}-\mu_{02}, s_{1}=2 \mu_{11}, c_{2}=\mu_{03}-3 \mu_{21}, s_{1}=\mu_{30}-3 \mu_{12}$

For non- symmetrical objects $\quad P_{x}=\frac{H_{1}}{H_{3}^{2}} \quad P_{y}=a \frac{H_{2}}{H_{3}^{3}}$

and symmetrical objects $s_{x}=\frac{c_{2} c_{3}+s_{2} s_{3}}{K} \quad s_{y}=\frac{s_{2} c_{3}-c_{2} s_{3}}{K} \quad c_{3}=c_{1}^{2}-s_{1}^{2} \quad s_{3}=2 s_{1} c_{1} \quad K=\frac{H_{1} H_{3}^{(3 / 2)}}{\sqrt{a}}$

This singularity is essentially due to the use of central moments (computed with respect to the object centroid). In the sequel, we will provide features computed from Legendre moments suitable in both cases. At this aim, let us modify the general form of two-dimensional Legendre central moments in equation (9) by substituting the coordinates of the object centroid $\mathrm{x}_{\mathrm{c}}$ and $\mathrm{y}_{\mathrm{c}}$ by $\left(\mathrm{x}_{\mathrm{c}}-\mathrm{x}_{\mathrm{s}}\right)$ and $\left(\mathrm{y}_{\mathrm{c}}-\mathrm{y}_{\mathrm{s}}\right)$ respectively, where

$$
x_{s}=\rho\left[\frac{\lambda_{20}}{\lambda_{00}}-5 x_{c} \frac{\lambda_{10}}{\lambda_{00}}+\left(\frac{15 x_{c}^{2}}{2}+\frac{5}{2}\right)\right]^{\frac{1}{2}} \quad y_{s}=\sigma\left[\frac{\lambda_{02}}{\lambda_{00}}-5 y_{c} \frac{\lambda_{01}}{\lambda_{00}}+\left(\frac{15 y_{c}^{2}}{2}+\frac{5}{2}\right)\right]^{\frac{1}{2}}
$$

and where the shift factors $\rho$ and $\sigma$ can take any non-zero values. $x_{\mathrm{s}}$ and $\mathrm{y}_{\mathrm{s}}$ are defined such that equation (9) preserves the translation invariance and gives non-zero values for all odd-orders central moments of symmetrical and non-symmetrical objects. The simplest invariants consist then of a combination of second order and third order Legendre moments:

$$
r_{1}=\frac{\Delta^{3}}{a\left[\left(\varphi_{03}-3 \varphi_{21}\right)^{2}+\left(\varphi_{30}-3 \varphi_{12}\right)^{2}\right]^{2}} \quad r_{2}=\frac{\left(\varphi_{20} \varphi_{02}-\varphi_{11}^{2}\right)^{3}}{a^{2} \Delta_{1}^{2}}
$$

In order to control the six degrees of freedom of the camera, we will thus use the following 6dimensional vector of visual features $s_{\lambda}=\left[\begin{array}{llllll}x_{n} & y_{n} & a_{n} & r_{1} & r_{2} & \beta\end{array}\right]$. These features are suitable for symmetrical and non-symmetrical objects when computed from Legendre moments but they are suitable only for non-symmetrical objects when computed from geometric moments. This is an important advantage of our proposal. In the sequel, the visual features $s_{m}=\left[\begin{array}{lllll}x_{n} & y_{n} & a_{n} & s_{x} & s_{y}\end{array}\right]$ for symmetrical objects and $\mathrm{s}_{\mathrm{m}}=\left[\begin{array}{llllll}\mathrm{x}_{\mathrm{n}} & \mathrm{y}_{\mathrm{n}} & \mathrm{a}_{\mathrm{n}} & \mathrm{P}_{\mathrm{x}} & \mathrm{P}_{\mathrm{y}} & \Phi\end{array}\right]$ for non-symmetrical objects computed from geometric moments will be used to compare Geometrical and Legendre moments in the context of visual servoing. 


\section{Results}

In this section, we compare the new set of visual features $s_{\lambda}$ computed from Legendre moments with the classical set of visual features $s_{m}$ computed from Geometrical moments. Simulated results were carried out using the software VISP (VIsual Servoing Platform) [18]. The experimental results have been obtained with a 6-DOF eye-in-hand system. The moments have been computed using the method presented in [19]. The parameters of the object plane in the camera frame (in its desired position) are approximately $\mathrm{A}=\mathrm{B}=0$ and $\mathrm{C}=2$. A non-symmetrical object has been used to carry out our experimentations.

\section{Simulation results}

In this section, we exhibit a case where geometric moments are highly sensitive to noise while Legendre moments give satisfactory results. We consider a symmetrical target composed of four planar points with the following coordinates in the object frame: $(-0.10,0.10,0.0),(0.10,0$. $10,0.0),(0.10,-0.10,0.0)$ and $(-0.10,-0.10,0.0)$ and non-symmetrical target composed of four planar points with the following coordinates in the object frame: $(-0.10,0.10,0.0),(0.10$, $0.10,0.0),(0.35,-0.45,0.0)$ and $(-0.01,-0.25,0.0)$ (see Fig.1). The simulation results using $\mathrm{s}_{\lambda}$ are depicted in Fig. 2 and in Fig. 4 while the simulation results using $\mathrm{s}_{\mathrm{m}}$ are depicted in Fig.3 and in Fig.5. We can see the improvements brought by the proposed features since they allow to obtain the same exponential decoupled decrease for the visual features and for the components of the camera velocity. By analyzing this simulation, we can see that Legendre moments are more robust to noise than geometric moments.
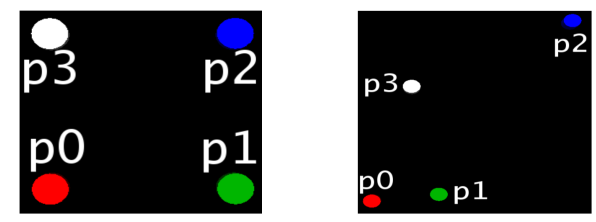

Figure.1. The target considered in the simulations. Left: symmetrical image; right: non-symmetrical image.

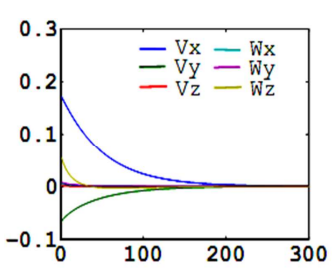

Figure.2. Simulation results with symmetrical image using $s_{\lambda}$. Left: camera velocity (in $\mathrm{m} / \mathrm{s}$ and $\mathrm{rad} / \mathrm{s}$ ); right: visual features errors.
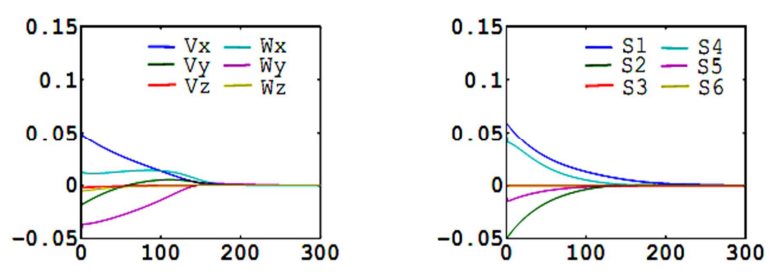

Figure.3. Simulation results with symmetrical image using $\mathrm{s}_{\mathrm{m}}$. left: camera velocity components (in $\mathrm{m} / \mathrm{s}$ and $\mathrm{rad} / \mathrm{s}$ ); right: visual features errors. 

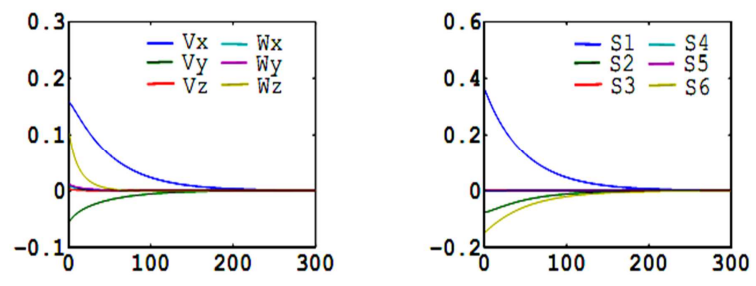

Figure.4. Simulation results with nonsymmetrical image using $\mathrm{s}_{\lambda}$. Left: camera velocity (in $\mathrm{m} / \mathrm{s}$ and $\mathrm{rad} / \mathrm{s}$ ); right: visual features errors.
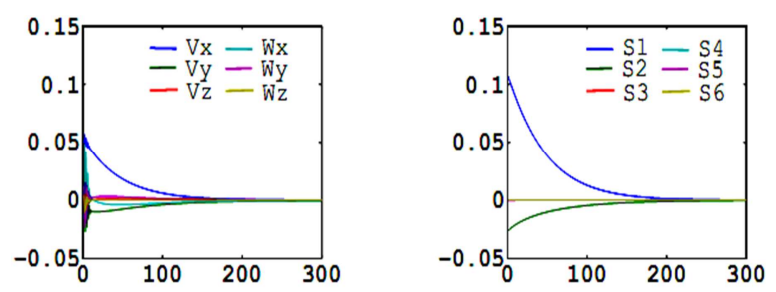

Figure.5. Simulation results with nonsymmetrical image using $\mathrm{s}_{\mathrm{m}}$. left: camera velocity components (in $\mathrm{m} / \mathrm{s}$ and $\mathrm{rad} / \mathrm{s}$ ); right: visual features errors.

The numerical value of the interaction matrix for the target computed at the desired position has the following form

$$
L_{s_{\lambda} / s_{h}=s_{i}^{*}}^{\|}=\left[\begin{array}{cccccc}
-1 & 0 & 0 & 0 & -0.5 & 0 \\
0 & -1 & 0 & 0.5 & 0 & 0 \\
0 & 0 & -1 & 0 & 0 & 0 \\
0 & 0 & 0 & 0.10 & -0.10 & 0 \\
0 & 0 & 0 & -0.02 & 0.02 & 0 \\
0 & 0 & 0 & 0.23 & 0.24 & -2
\end{array}\right] \quad L_{s_{m} / s_{m}=s_{m}^{*}}^{\|}=\left[\begin{array}{cccccc}
-1 & 0 & 0 & 0 & -0.5 & 0 \\
0 & -1 & 0 & 0.5 & 0 & 0 \\
0 & 0 & -1 & 0 & 0 & 0 \\
0 & 0 & 0 & 0.10 & 0 & 0 \\
0 & 0 & 0 & 0 & 0.10 & 0 \\
0 & 0 & 0 & 0 & 0 & -1
\end{array}\right]
$$

As expected, the interaction matrix related to Legendre moments and Geometric moments are blocs triangular with main term around the diagonal.

\section{Experimental results}

The first experiment (ER1) concerns a pure translational motion toward the object. The gain $\lambda$ have been set to 0. 5. The results are depicted in Fig.6. and Fig.7. The last experiment (ER2) concerns a generic motion around and along the three axes of the camera frame (refer to Fig. 8 and Fig. 9). In all cases the features $\mathrm{s}_{\lambda}$ allows to obtain very satisfactory results (exponential decrease of the errors without any oscillations). As expected, we note also that the results obtained with geometrical moments are more corrupted by noises than the results obtained using Legendre moments.
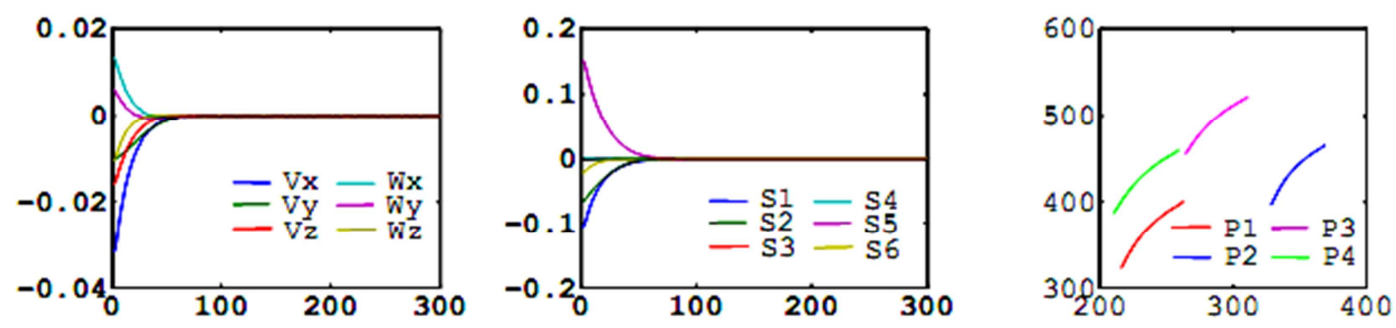

Fig.6. ER1 using $\mathrm{s}_{\lambda}$. Left: camera velocity (in $\mathrm{m} / \mathrm{s}$ and $\mathrm{rad} / \mathrm{s}$ ), middle: visual features errors, right: image points trajectories. 

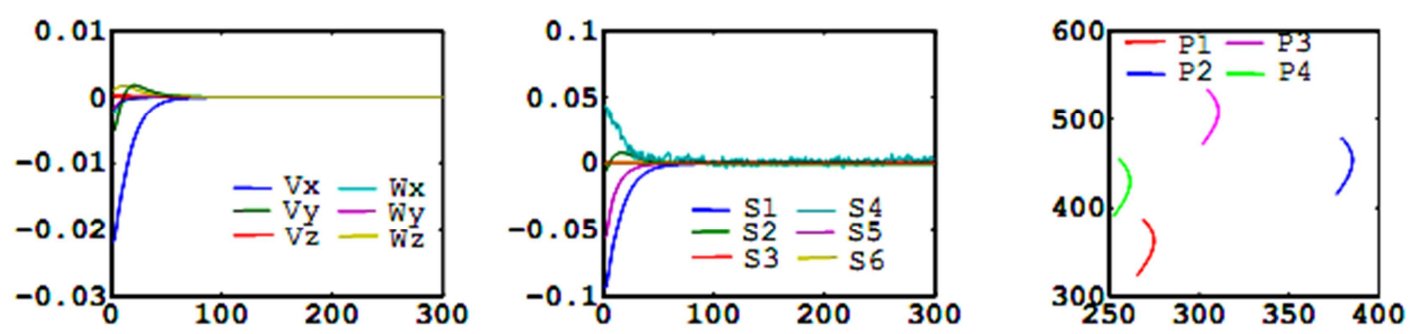

Fig.7. ER $1 \mathrm{~s}_{\mathrm{m}}$. Left: camera velocity (in $\mathrm{m} / \mathrm{s}$ and $\mathrm{rad} / \mathrm{s}$ ), middle: visual features errors, right: image points trajectories.
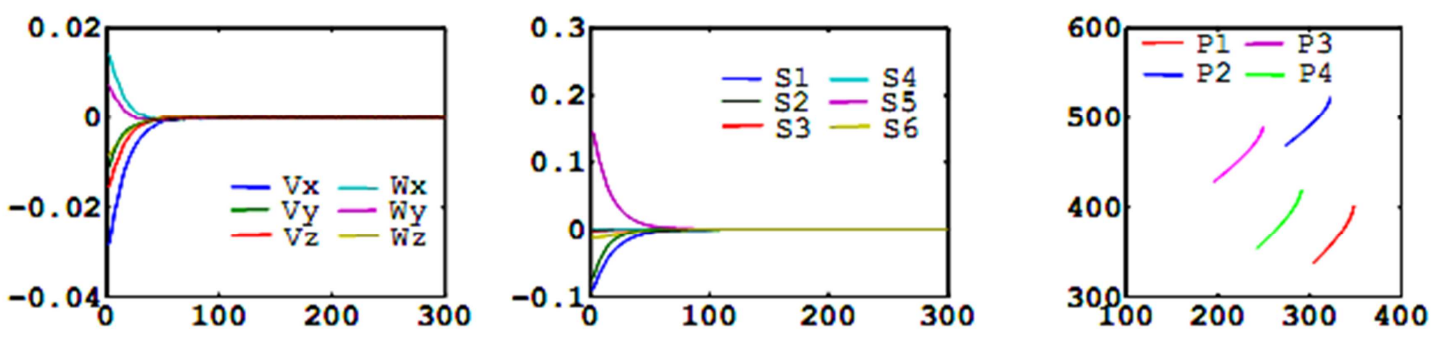

Fig.8. ER2 $\mathrm{s}_{\lambda}$. Left: camera velocity components (in $\mathrm{m} / \mathrm{s}$ and $\mathrm{rad} / \mathrm{s}$ ), middle: visual features errors, right: image points trajectories.
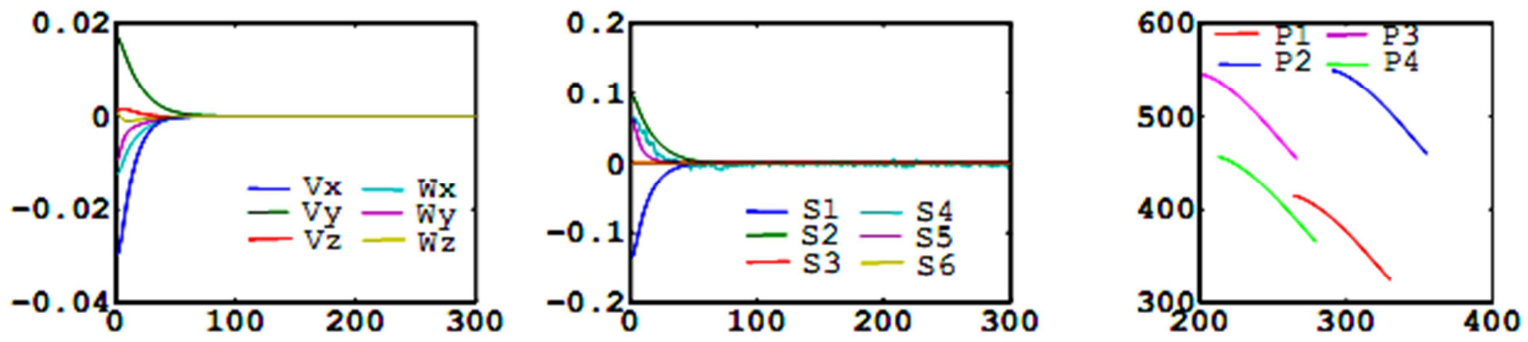

Fig.9. ER2 $\mathrm{s}_{\mathrm{m}}$. Left: camera velocity (in $\mathrm{m} / \mathrm{s}$ and $\mathrm{rad} / \mathrm{s}$ ), middle: visual features errors, right: image points trajectories.

\section{Conclusion}

In this paper, a class of orthogonal moments (namely Legendre moments) have been exploited to improve the behavior of moment-based control schemes. The descriptive form of the interaction matrix related to any Legendre moment computed from a set of points has been obtained and six visual features have been selected to design a partially-decoupled control scheme. Experimental results have shown that the new set of visual features give better results than a similar set of features computed from geometric moments. We are currently working on extending this work to complex closed contours. 


\section{References}

[1] Ming-Kuei Hu. Visual pattern recognition by moment invariants. Information Theory, IRE Transactions on, 8(2):179-187, 1962.

[2] M. R. Teague. Image analysis via the general theory of moments. Journal of the Optical Society of America, 70 Issue 8:920-930, 1980.

[3] Alireza Khotanzad and Yaw Hua Hong. Invariant image recognition by zernike moments. IEEE Trans. Pattern Anal. Mach. Intell.

[4] R. Mukundan, S. H. Ong, and Poh Aun Lee. Image analysis by tchebyshev moments. IEEE Transactions on Image Processing.

[5] R. Mukundan. Some computational aspects of discrete orthonormal moments. IEEE Transactions on Image Processing.

[6] C.H. Teh and R.T. Chin. On image analysis by the methods of moments. IEEE Transactions on Pattern Analysis and Machine Intelligence, 10:496-513, 1988.

[7] Chee-Way Chong, P. Raveendran, and R. Mukundan. The scale invariants of pseudo-zernike moments. Pattern Anal. Applications, pages 176-184, 2003.

[8] Chee-Way Chong, P. Raveendran, and R. Mukundan. Translation and scale invariants of Legendre moments. Pattern Recognition, 37(1):119-129, 2004.

[9] Khalid M. Hosny. Refined translation and scale Legendre moment invariants. Pattern Recogn. Lett., 31:533-538, May 2010.

[10] Khalid M. Hosny. New set of rotationally Legendre moment invariants. International Journal of Electrical, Computer, and Systems Engineer-ing, 4:533-538, May 2010.

[11] F. Chaumette. Image moments: a general and useful set of features for visual servoing. IEEE Trans. on Robotics, 20(4):713-723, August 2004.

[12] O. Tahri and F. Chaumette. Application of moment invariants to visual servoing. In IEEE Int. Conf. on Robotics and Automation, ICRA'03, volume 3, pages 4276-4281, Taipeh, Taiwan, May 2003.

[13] O. Tahri and F. Chaumette. Point-based and region-based image moments for visual servoing of planar objects. IEEE Trans. On Robotics, 21(6):1116-1127, December 2005.

[14] K.R.Ramakrishnan R.Mukundan. Moment functions in image analysis: Theory and applications. In World Scientific Publishing Co Pte Ltd., Singapore, page 165, September 1998.

[15] S. S. Reddi. Radial and angular moments invariants for image identification. IEEE Trans. Pattern Anal. Mach. Intell.

[16] Y.S. Abu Mostafa and D. Psaltis. Recognitive aspects of moment invariants. PAMI, 6(6):698706, November 1984.

[17] P.I. Corke and S. Hutchinson. A new partitioned approach to image-based visual servo control. IEEE Transactions on Robotics and Automation, 17:507515, aug 2001.

[18] E. Marchand, F. Spindler, and F. Chaumette. Visp for visual servoing: a generic software platform with a wide class of robot control skills. IEEE Robotics and Automation Magazine, 12(4):40-52, December 2005.

[19] C. Steger. On the calculation of arbitrary moments of polygons. Technical Report FGBV9605, Technische UniversitM, oct 1996. 